\title{
Comparison of 2D and 3D Clustering on Short-Axis Magnetic Resonance Images of the Left Ventricle
}

\section{Introduction:}

A comparative study is performed between segmentation results of the left ventricle endo-cardial boundary (inner wall) using a 2D and 3D clustering approach.

Segmentation of the endo-cardial boundary is an important process in the evaluation of the left ventricle cavity volume, used to measure heart function.

The ejection fraction is an important measurement for the early prognosis and treatment monitoring of many types of Cardiovascular Disease (CVD).

$$
E F=\frac{V\left(t_{D}\right)-V\left(t_{S}\right)}{V\left(t_{D}\right)}
$$

\section{Procedure:}

The volume images are first smoothed using a nonlinear adaptive filter to remove unwanted noise signals while maintaining the strong edge features in each slice of the volume.

The image is clustered using an unsupervised $k$ means clustering technique.

- The 2D algorithm joins clusters with similar intensity signals on each slice in the short-axis plane

- The 3D algorithm joins the clusters through the short axis volume from the base of the ventricle to its apex.

The left ventricle is automatically located using the least squares approximation to a circle and an ellipsoid for the 2D and 3D case respectively.

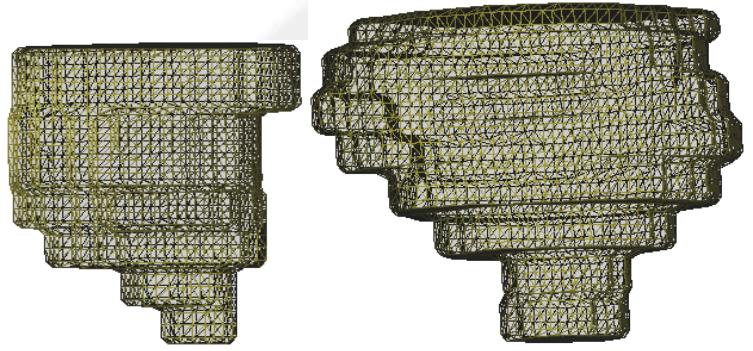

M. Lynch, O. Ghita, P.F. Whelan

Vision Systems Group, Dublin City University

Results:
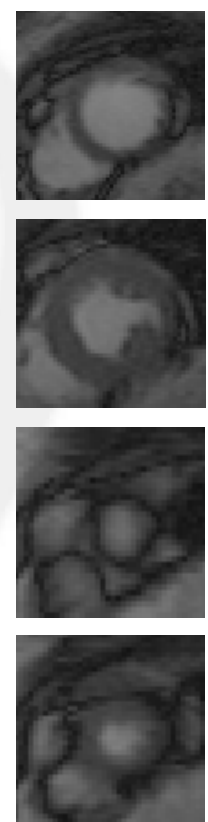

Figure 1: Column 1 is the original image, columns 2 and 3 show the results after $2 \mathrm{D}$ and $3 \mathrm{D}$ clustering and localization of the left ventricle.

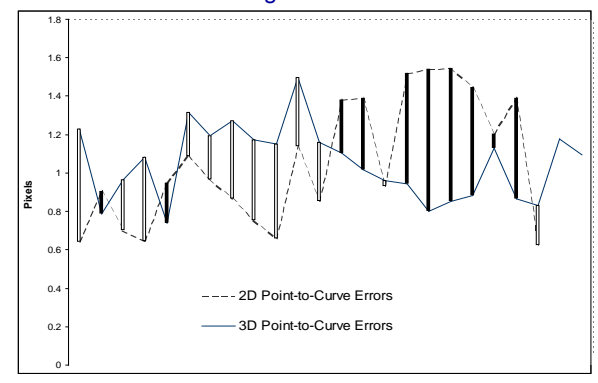

Figure 2: Standard deviation of the point-to-curve measures for each volume with error between the 2D and 3D method

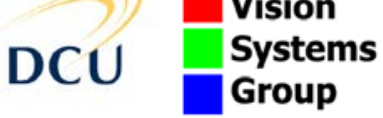

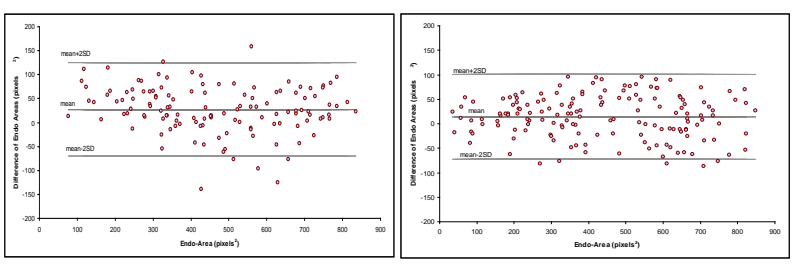

Figure 3: Bland-Altman plot for the automatic segmentation against the manual segmentation for the 2D case (left) and the 3D case (right).

The table below shows the area analyis of the endocardium using the automatic approach against the manual approach. RMS and pixel error are \pm 1 standard deviation.

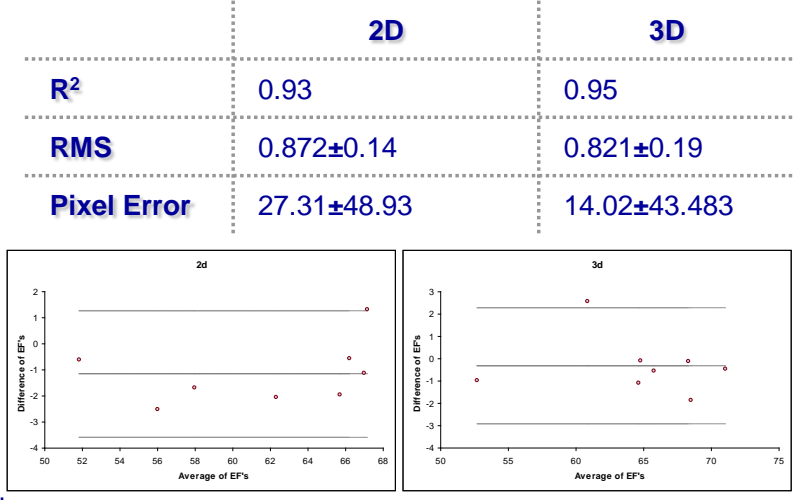

Figure 4: Bland-Altman plot the calculation of the ejection fraction for both the $2 \mathrm{D}$ case (left) and the 3D case (right)

With respect to volume analysis, the ejection fraction, results has an $\mathrm{R}^{2}$ value of 0.96 and 0.95 and percent error of $-1.15 \pm 1.21 \%$ and $-0.31 \pm 1.30 \%$ for the $2 \mathrm{D}$ and $3 \mathrm{D}$ respectively.

\section{Conclusions:}

In conclusion, the 3D clustering technique gives more accurate segmentation results and more accurate and robust results closer to the apex.

Ejection fraction is less effected as apical volume is small when compared to the overall volume. 